\title{
Características psicométricas de un cuestionario de expectativas hacia la educación virtual en estudiantes universitarios durante la pandemia COVID-19
}

\author{
Karla Lobos ${ }^{1}$, Claudio Bustos ${ }^{2}$, Rubia C. Cobo-Rendón ${ }^{1 *}$ y Nataly V. Cisternas ${ }^{1}$ \\ (1) Laboratorio de Investigación e Innovación Educativa, Dirección de Docencia, Universidad de Concepción, \\ Concepción-Chile (correo-e: karlalobos@udec,cl, rubiacobo@udec,cl, ncisternas@udec,cl) \\ (2) Departamento de Psicología, Facultad de Ciencias Sociales, Universidad de Concepción, Concepción-Chile \\ (correo-e: clbustos@udec,cl)
}

* Autor a quien debe ser dirigida la correspondencia.

Recibido May. 4, 2021; Aceptado Jun. 30, 2021; Versión final Ago. 26, 2021, Publicado Feb. 2022

\begin{abstract}
Resumen
El propósito de este estudio es diseñar un cuestionario de expectativas hacia la educación virtual para estudiantes universitarios durante la pandemia por COVID-19. Debido a la pandemia, las universidades tuvieron el desafío de proveer programas de formación virtual adaptados a las expectativas de los estudiantes. Participaron 8265 estudiantes (4627 mujeres) de una universidad de Chile con una media de edad de 21,53 ( $\mathrm{DE}=2,87), 33,1 \%$ pertenecían a primer año académico. Se construyó un cuestionario a partir del análisis de la literatura científica y se realizó la validación por juicio de expertos. Se realizaron estimaciones de validez con análisis factoriales exploratorio y confirmatorio. Los resultados muestran un ajuste adecuado y un modelo con 6 factores derivados en una solución jerárquica. Los coeficientes de consistencia interna fueron adecuados. En conclusión, el cuestionario de expectativas hacia la educación virtual para universitarios es válido y confiable para la medición de este constructo en la educación superior chilena.
\end{abstract}

\section{Psychometric characteristics of a virtual education expectations questionnaire for university students during the COVID-19 pandemic}

\begin{abstract}
The primary aim of this research study was to design a questionnaire that measures expectations towards online education for university students during the COVID-19 pandemic. Due to the COVID-19 pandemic, university authorities faced the challenge of providing online training programs that were specifically adapted to meet student expectations. In total, 8265 students $(4627$ women) from a Chilean university participated in the study. The mean age was 21.53 years $(S D=2.87)$ and $33.1 \%$ of the students were in their first academic year. A questionnaire was designed based on a scientific literature review and was validated by expert judgment. Validation estimates with exploratory and confirmatory factor analyses were performed. The results showed an adequate fit and a model with six derived factors in a hierarchical solution. Internal consistency coefficients were adequate. In conclusion, the virtual education expectations questionnaire for university students is valid and reliable for Chilean higher education.
\end{abstract}




\section{INTRODUCCIÓN}

Con la finalidad de disminuir la velocidad de contagios entre los y las estudiantes, una de las consecuencias de la pandemia generada por el COVID-19 a inicio del 2020, fue el cierre de las instituciones de Educación Superior. Para continuar los procesos de formación profesional de los y las jóvenes, las universidades se han visto forzadas a continuar sus clases en modalidad virtual (Velavan y Meyer, 2020). La educación virtual no es algo nuevo y su implementación en las instituciones de Educación Superior ha sido de forma progresiva, es debido a la pandemia por el COVID-19, que el sector educativo ha tenido que adaptarse con mayor velocidad a esta modalidad. Las universidades han encontrado en la educación virtual un elemento clave para generar nuevos espacios de aprendizaje, fomentando el uso de diversas plataformas y aplicaciones web que facilitan a los y las estudiantes alcanzar los objetivos de aprendizaje (Crisol-Moya et al., 2020). Al mismo tiempo que han permitido incorporar nuevas formas de enseñar e investigar, para la mejora de los procesos educativos (Nieto-Sánchez, 2018).

La Educación virtual es una estrategia de alto impacto en la mejora de la cobertura, pertinencia y calidad educativa debido a sus características multimediales, hipertextuales e interactivas (Crisol-Moya et al., 2020). Este tipo de educación se desarrolla dentro de un entorno tecnológico de aprendizaje; una aplicación informática diseñada para facilitar la comunicación pedagógica entre quienes participan de un proceso educativo, desde modalidades completamente a distancia, presencial, o de naturaleza mixta (Santamaría y Calvo, 2012).

Dentro de los entornos de educación virtual, podemos encontrar los sistemas de gestión del aprendizaje o Learning Management System (LMS) (Salvat, 2018). Los LMS son softwares diseñados para dar soporte a los cursos que incluyen elementos de administración, comunicación sincrónica y asincrónica, compartición multimedia, evaluación y seguimiento de actividades (Osorio-Gómez y Duart, 2012). Este tipo de programas presentan varios beneficios: primero, generan altos grados de satisfacción en cuanto al papel autónomo y flexible de los y las estudiantes. Segundo, permiten el desarrollo de competencias digitales y la colaboración e interactividad entre las personas que aprenden y las personas que enseñan, todo dentro de una modalidad de clases sin restricciones asociadas al tiempo y espacio (Lorenzo-Lledó et al., 2018).

Una revisión sistemática que analizó investigaciones publicadas entre 2010-2018 (Rodrigues et al., 2019), indica que el aprendizaje electrónico o e-learning presenta características importantes que lo definen, estas son: 1) las características del curso; incluyen la evaluación de la calidad de los materiales de aprendizaje, del entorno virtual y la coherencia de los componentes curriculares con las competencias por alcanzar al finalizar la formación; 2) las características relacionadas con los resultados de los aprendizajes, que incluyen aspectos vinculados al logro de las competencias y del conocimiento conceptual, metódico y social y 3) las características relacionadas con las experiencias de interacción de los estudiantes con sus pares y profesores; que incluyen la comunicación y retroalimentación de los docentes sobre los logros de los objetivos, el fomento de la motivación y del compromiso de los y las estudiantes en el curso (Rodrigues et al., 2019).

En el contexto de la pandemia por el COVID-19, el éxito de la enseñanza en la educación virtual radica en que tanto docentes como estudiantes puedan ser capaces de adaptarse e involucrarse ampliamente en las plataformas de LMS, aulas virtuales, foros y chats que estas ofrecen (Ramírez-Anormaliza, et al., 2017).=Uno de los obstáculos que pudieran presentarse en los y las estudiantes, se relaciona con las expectativas generadas sobre los cursos e-learning; intereses que pudieran acercarse o alejarse al tipo de contenido y experiencia que estos cursos ofrecen para los y las participantes (Rodrigues et al., 2019). Las expectativas son consideradas como predictores del éxito y alto rendimiento académico en estudiantes universitarios (Paechter et al., 2010). El diseño de cursos en línea debe ajustarse a las características psicosociales (compromiso, satisfacción, motivación, autodisciplina, autoeficacia y experiencia previa) de los y las estudiantes para así poder satisfacer sus necesidades y expectativas (Rodrigues et al., 2019).

Uno de los modelos teóricos con mayor aceptación para la evaluación de las expectativas de los y las estudiantes en los cursos e-learning es el Modelo de Aceptación Tecnológica (Revythi y Tselios, 2019). Este modelo busca examinar y explicar cómo y por qué los y las estudiantes aceptan o rechazan una tecnología. Se sustenta en la Teoría de la Acción Razonada (Ajzen y Fishbein, 1980), la cual describe que el comportamiento está influenciado por las actitudes, creencias y evaluación del comportamiento de los usuarios (Davis, 1993). Según este modelo existen factores claves en la evaluación de la aceptación como, por ejemplo, la facilidad de uso percibida, la utilidad percibida, la actitud hacia el uso y la presencia de variables externas como el diseño de la plataforma (Davis, 1993; Revythi y Tselios, 2019).

Con respecto a la medición de las expectativas de los y las estudiantes sobre los cursos en línea. Una revisión sistemática sobre investigaciones publicadas desde 1980 hasta 2019 en el área de la Educación de las Ciencias de la Salud (Regmi y Jones, 2020), refiere que, para la medición de las expectativas existen factores 
internos y externos vinculados al aprendizaje electrónico. En este caso, las expectativas de los y las estudiantes pueden ser un obstáculo o facilitador en el uso de las plataformas en línea (Regmi y Jones, 2020), dentro de los factores internos se encuentran, los referidos al nivel de compromiso, motivación, autoeficacia e interacciones entre estudiantes y docentes (Regmi y Jones, 2020).

En este caso, la autoeficacia es definida como la creencia personal que una persona tiene sobre el dominio en una actividad (Bandura, 2012). Por la cualidad de ser una creencia sobre un dominio específico, existen diversos tipos de autoeficacia evaluados en el aprendizaje en línea, por ejemplo, la autoeficacia tecnológica, creencia sobre las habilidades y destrezas para tener éxito con una tarea relacionada con la tecnología (Cai et al, 2017). Este tipo de creencias proporcionan a los y las estudiantes facilidades para que estos usen en mayor o menor medida los sistemas de aprendizaje en línea, siendo consideradas como factores que incrementan la intención del aprendizaje electrónico (Al-Rahmi et al., 2018). Otro factor interno, relevante con respecto a las expectativas en el uso de las plataformas de aprendizaje en línea, son las oportunidades que poseen los y las estudiantes de interactuar con sus pares y con él o la docente. La literatura refiere que existen varias formas de interacción en línea como lo son, las relaciones entre estudiante-estudiante, estudianteinstructor, contenido-estudiante, interfaz-estudiante e interacción indirecta (Moore, 1989). La evidencia empírica refiere que la relaciones entre pares y con docentes en estas plataformas, es uno de los factores principales para incrementar la motivación en el uso de estas (Ghavifekr y Mahmood, 2017).

En el caso de los factores externos que se vinculan a las expectativas de los y las estudiantes, es posible identificar elementos como: la estructura del curso, el diseño pedagógico, la claridad del propósito y meta, política de gestión educativa, paradigmas educativos, uso de tecnología en educación, capacitaciones basadas en evidencia, entre otros (Regmi y Jones, 2020). Investigaciones refieren que la satisfacción de los estudiantes durante las experiencias de educación en línea varía de acuerdo con la calidad del contenido instruccional, las formas de comunicación y el como se desarrollan los procesos de enseñanza (llgaz y Gülbahar, 2015). Considerar las expectativas, necesidades y preferencias de los y las estudiantes, se ha convertido en aspectos críticos para la evaluación de asignaturas. Cuando los y las estudiantes encuentran que los sistemas de aprendizaje son fáciles y útiles para su propio aprendizaje presentan una actitud positiva hacia el aprendizaje electrónico beneficiando su experiencia formativa (Regmi y Jones, 2020). En otros estudios se ha demostrado que las expectativas de los estudiantes sobre la educación en línea favorecen la permanencia de los aprendices. Existe una relación entre las expectativas de los estudiantes y sus percepciones acerca del tiempo y del nivel de dificultad que deben realizar para lograr el éxito en este tipo de modalidad. En la medida que los estudiantes tienen expectativas positivas frente a los escenarios de educación en línea, mayor será la probabilidad de elegir este tipo de formación.

Con base a los planteamientos teóricos, la evidencia empírica encontrada y la situación actual a la que se enfrentan todos los integrantes de las instituciones de Educación Superior debido al COVID-19. Se consideran como elementos relevantes para la medición de las expectativas, las creencias de autoeficacia tecnológica, la calidad de la relación con pares y la calidad de la relación con docentes, la percepción de compromiso institucional y la percepción de la calidad del aprendizaje y de la enseñanza. Todos estos elementos han sido asociados al incremento en el uso de las plataformas digitales, en la percepción del aprendizaje y en la satisfacción de los y las estudiantes (Roig-Vila et al., 2015). Los instrumentos existentes sobre educación virtual son escasos y abordan aspectos de satisfacción, utilidad y facilidad percibida en el uso de distintas herramientas tecnológicas educativas y plataformas de gestión del aprendizaje (Al-Maroof et al., 2021). Del mismo modo, existen instrumentos que evalúan el nivel de competencia y/o conocimiento docente para la integración tecnológica (Scott y Nimon, 2020) pero no como está se lleva a cabo en sus distintos componentes.

Así mismo, este cuestionario se enfoca en la evaluación de las expectativas en el escenario de la educación remota de emergencia producida por la pandemia por COVID-19. Los efectos sociales y psicológicos generados por la pandemia han creado la necesidad de construir instrumentos de medición específicos para este contexto (De los Ríos et al., 2020; Mamani et al., 2020). Contar con un cuestionario válido y confiable permitirá ayudar a las autoridades de las universidades en identificar y comprender los aspectos que intervinieron de manera significativa en la experiencia de los y las estudiantes durante la educación remota de emergencia producida por la pandemia, aspectos que son necesarios detectar a tiempo para mejorar la experiencia educativa y minimizar el riesgo de fracaso de los estudiantes.

De los antecedentes revisados, se plantea la necesidad de contar con un instrumento que permita evaluar las expectativas hacia la educación virtual en estudiantes universitarios identificando anticipadamente creencias que pudieran actuar como predictores de dificultades en los procesos de enseñanza y aprendizaje en esta modalidad. Por esta razón, es necesario contar con un instrumento especializado que permita evaluar las expectativas hacia la educación virtual en estudiantes universitarios. Esta investigación tiene por objetivo diseñar y validar el cuestionario de expectativas hacia la educación virtual en estudiantes universitarios 
durante la pandemia por COVID-19. Todo esto, con la finalidad de proporcionar herramientas que permitan la investigación de estos aspectos bajo el escenario producido por la COVID-19 y el desarrollo e implementación de intervenciones que beneficien los procesos de adaptación y de enseñanza-aprendizaje virtual en la Educación Superior.

\section{MÉTODO}

El presente estudio corresponde a un diseño de tipo instrumental (Montero y León, 2007) con el objetivo de diseñar y validar un cuestionario de expectativas hacia la educación virtual en estudiantes universitarios durante la pandemia por COVID-19.

\section{Muestra}

Se analizaron las respuestas de 8,265 estudiantes de una universidad del sur de Chile (4,627 mujeres; 3,638 hombres), con edades comprendidas entre 18 y 49 años $(M=21.53 ; D E=2.87)$. Con respecto a la distribución de los y las estudiantes según áreas científicas, 9\% pertenecían a carreras de Ciencias Naturales, un $24 \%$ a carreras de Ingeniería y Tecnología, un 22\% a carreras de Ciencias Médicas y de la Salud, un 15\% a carreras de Ciencias Agrícolas, $13 \%$ a carreras de Ciencias Sociales y un $18 \%$ a carreras de Humanidades y Educación. La muestra $(n=8,265)$ se dividió en dos partes de forma aleatoria, estratificando por cohorte (2014 o menor y desde 2015 al 2020), facultad y género, para generar dos muestras que fueran relativamente similares en estas variables. En la primera sub-muestra $(n=4,132)$ se realizó un análisis factorial exploratorio en tanto que la segunda sub-muestra $(n=4,133)$, se aplicó el análisis factorial confirmatorio. El procedimiento para la selección de los y las participantes fue realizado a partir de un muestreo no probabilístico por conveniencia,

\section{Instrumentos}

El cuestionario de Expectativas hacia la Educación virtual en Educación Superior para estudiantes (CEEVESE) tiene como objetivo conocer las expectativas acerca de la educación virtual que poseen los estudiantes de educación superior. Está constituido por 35 ítems distribuidos en seis dimensiones del estudiante acerca de la educación virtual. En la tabla 1 se describen las dimensiones que componen el cuestionario.

\section{Procedimiento}

Para la construcción del instrumento, los y las autoras se basaron en la revisión de la literatura para desarrollar preliminarmente cinco dimensiones con 45 reactivos, 8 para cada dimensión, con la intención de disponer de un repertorio suficiente de preguntas para realizar una selección posterior de los mejores ítems. Las dimensiones preliminares eran: Compromiso Institucional, Calidad de los Procesos de Enseñanza Aprendizaje, Calidad de la relación con Pares, Calidad en la relación con Docentes y Autoeficacia percibida para la Educación virtual, La validez de contenido se estudió mediante el juicio de experto, seleccionando seis profesionales, bajo dos criterios: (1) grado de Magíster o Doctor, (2) producción científica vinculada a la educación universitaria, y (3) experiencia en educación e-learning o b-learning.

En los resultados entregados por las y los jueces expertos se sugieren cambios tanto en cuanto a las dimensiones como de los reactivos. Con respecto a dimensiones, se sugiere que la dimensión "Calidad de los Procesos de Enseñanza Aprendizaje" se divida en dos, una que aborde exclusivamente las expectativas acerca de cómo los y las estudiantes perciben que serán sus aprendizajes mediante el aula virtual y los resultados que espera alcanzar y, otra, que aborde aspectos de la enseñanza que son más propios de los procedimientos efectuados por el o la docente como el cumplimiento de las planificaciones, el respeto de los horarios asignados, la entrega de información sobre los cursos y la organización del tiempo para el cumplimiento de las actividades académicas. La primera aborda de manera directa expectativas sobre los aprendizajes, en la segunda, se abordan las condiciones que favorecen el aprendizaje, pero que son circundantes a él. De esta forma, se crean dos nuevas dimensiones: "Calidad de la Enseñanza" y "Calidad del Aprendizaje".

Con respecto a reactivos, la división de la dimensión de Calidad de los procesos de enseñanza y aprendizaje, en dos, implica una reducción de reactivos, quedando 3 ítems en la primera y 5 en la segunda de los 10 previamente estimados para una sola dimensión. Para la dimensión calidad de la enseñanza, se construyen 5 nuevos reactivos y para la dimensión calidad del aprendizaje, se construyen 3 , quedando ambas con 8 ítems cada una. El resto de las dimensiones sufrieron modificaciones en sus reactivos. La dimensión de Compromiso Institucional se reduce a 4 reactivos, dado que a juicio de las y los expertos, 3 de ellos no correspondía a este constructo y 1 poseía problemas de redacción. En la dimensión de Calidad en la relación 
con Pares se elimina 1 reactivo por considerar que no responderían a la dimensión teórica para la cual fue diseñada. En la dimensión Calidad de la relación con Docentes, 4 ítems fueron eliminados por considerar una redacción confusa y poco comprensible, y que dos de ellos ya estaban considerados en los reactivos restando, quedando un número de 4 ítems. Finalmente, en lo que refiere a la Autoeficacia percibida para la educación virtual, 2 reactivos fueron eliminados por no responder la dimensión teórica previamente establecida, quedando 6 reactivos. Uno de estos seis reactivos, se consideró que abordaba dos cualidades distintas, por lo que se procedió a su división, quedando conformada la dimensión por 8 reactivos.

Tabla 1: Descripción de las dimensiones del CEEVESE

\begin{tabular}{|c|c|c|}
\hline Dimensión & Descripción & Ítems y formato de respuesta \\
\hline $\begin{array}{l}\text { Compromiso } \\
\text { institucional }\end{array}$ & $\begin{array}{l}\text { Son las expectativas que posee el y la } \\
\text { estudiante acerca de si la universidad } \\
\text { brindará el apoyo necesario para el } \\
\text { desarrollo de la educación virtual (Hodges } \\
\text { et al., 2020). }\end{array}$ & $\begin{array}{l}4 \text { ítems respondidos por medio de una escala } \\
\text { Likert de } 5 \text { opciones de respuesta ( } 1=\text { Muy en } \\
\text { desacuerdo a } 5=\text { Muy de acuerdo). }\end{array}$ \\
\hline $\begin{array}{l}\text { Calidad de los } \\
\text { procesos de } \\
\text { enseñanza } \\
\text { aprendizaje }\end{array}$ & $\begin{array}{l}\text { Hace referencia a cómo él y la estudiante } \\
\text { percibe que serán sus aprendizajes } \\
\text { mediante el aula virtual y los resultados } \\
\text { que espera alcanzar (Crisol-Moya et al., } \\
2020 \text { ). }\end{array}$ & $\begin{array}{l}8 \text { ítems, de los cuales } 6 \text { son respondidos con } 5 \\
\text { opciones de respuestas y el resto con una escala } \\
\text { de tres opciones de respuesta ( } 1=\text { Peor que en la } \\
\text { clase tradicional / presencial, } 2=\text { Igual que en la } \\
\text { clase tradicional / presencial y } 3=\text { Mejor que en la } \\
\text { clase tradicional / presencial). }\end{array}$ \\
\hline $\begin{array}{l}\text { Calidad en la } \\
\text { planificación de la } \\
\text { enseñanza }\end{array}$ & $\begin{array}{l}\text { Busca evaluar las expectativas de los y las } \\
\text { estudiantes en torno a la forma de trabajo } \\
\text { y procedimientos que sus docentes } \\
\text { desarrollarán en aula virtual (Rodrígues et } \\
\text { al., 2019). }\end{array}$ & $\begin{array}{l}7 \text { ítems respondidos por medio de una escala } \\
\text { Likert de } 5 \text { opciones de respuesta ( } 1=\text { Muy en } \\
\text { desacuerdo a } 5=\text { Muy de acuerdo). }\end{array}$ \\
\hline $\begin{array}{l}\text { Calidad de la } \\
\text { relación con pares }\end{array}$ & $\begin{array}{l}\text { Tiene como finalidad evaluar las } \\
\text { expectativas de los y las estudiantes en } \\
\text { torno a si el aula virtual favorecerá o no la } \\
\text { relación social académica con pares } \\
\text { (Ghavifekr y Mahmood, 2017). }\end{array}$ & $\begin{array}{l}7 \text { ítems de los cuales } 5 \text { son respondidos por } \\
\text { medio de una escala con cinco opciones de } \\
\text { respuesta y dos con una escala de tres opciones } \\
\text { (1= Peor que en la clase tradicional / presencial, } \\
2=\text { Igual que en la clase tradicional / presencial y } \\
3=\text { Mejor que en la clase tradicional / presencial). }\end{array}$ \\
\hline $\begin{array}{l}\text { Calidad de la } \\
\text { relación con } \\
\text { docentes }\end{array}$ & $\begin{array}{l}\text { Tiene como objetivo la evaluación de las } \\
\text { expectativas de los y las estudiantes en } \\
\text { torno a si el aula virtual favorecerá o no la } \\
\text { relación social académica con sus } \\
\text { docentes (Ghavifekr y Mahmood, 2017). }\end{array}$ & $\begin{array}{l}4 \text { ítems, de los cuales tres son respondidas por } \\
\text { medio de una escala de tipo Likert con } 5 \\
\text { alternativas de respuesta y uno con una escala de } \\
\text { tres opciones de respuesta ( } 1=\text { Peor que en la } \\
\text { clase tradicional / presencial, } 2=\text { Igual que en la } \\
\text { clase tradicional / presencial y } 3=\text { Mejor que en la } \\
\text { clase tradicional / presencial). }\end{array}$ \\
\hline $\begin{array}{l}\text { Autoeficacia } \\
\text { percibida para la } \\
\text { educación virtual }\end{array}$ & $\begin{array}{l}\text { Hace referencia a la creencia de qué tan } \\
\text { capaz se percibe el y la estudiante de } \\
\text { poder hacer frente a los desafíos de la } \\
\text { educación en línea y responder con éxito a } \\
\text { los compromisos académicos (Cai et al, } \\
\text { 2017). }\end{array}$ & $\begin{array}{l}7 \text { ítems de los cuales seis son respondidos por } \\
\text { medio de una escala Likert de } 5 \text { opciones de } \\
\text { respuesta ( } 1=\text { Muy en desacuerdo a } 5=\text { Muy de } \\
\text { acuerdo). }\end{array}$ \\
\hline
\end{tabular}

El instrumento, en su versión final, quedo conformado por 35 reactivos, con 4 ítems para Compromiso Institucional, 8 ítems para Calidad de los Procesos Aprendizaje, 7 para Calidad de los Procesos de Enseñanza, 7 para Calidad de la relación con pares, 4 para Calidad de la relación con docentes y 7 para Autoeficacia percibida para la educación virtual. La versión final fue aplicada en forma piloto a 31 estudiantes, con el fin de evaluar la claridad de las instrucciones y la pertinencia del lenguaje. En esta aplicación no se reportaron dificultades en la redacción ni comprensión de sus distintos apartados.

Tras definir la estructura final del instrumento, se traspasó a un formato de formulario electrónico. La aplicación del cuestionario se realizó vía correo electrónico institucional a toda la población estudiantil de la universidad participante. El texto del correo electrónico especificaba los objetivos y alcances de la investigación y se invitaba a los y las estudiantes a participar accediendo a un enlace en donde antes de poder dar respuesta a las preguntas se le solicitaba la firma de consentimiento informado. La convocatoria para la participación en la investigación fue enviada en una sola oportunidad. El formulario se mantuvo abierto por un periodo de 2 semanas durante el mes de abril 2020, cuando los estudiantes universitarios participantes se encontraban iniciando su semestre académico en la modalidad de educación remota de emergencia. El tiempo de respuesta en promedio fue de 14 minutos. 


\section{Procedimientos para la obtención y el análisis de los datos}

El análisis consideró en primer lugar el análisis descriptivo de la muestra, para luego realizar el análisis factorial exploratorio y confirmatorio en las muestras correspondientes. Finalmente, se realizó el análisis de confiabilidad sobre la muestra completa y se analizó la distribución de las escalas resultantes del análisis factorial, estableciendo diferencias por sexo, facultad, cohorte y experiencia, usando pruebas no paramétricas. Todos los análisis fueron realizados usando software $\mathrm{R}$ (versión 3,6 ), para proceder con los análisis, los ítems de 1 a 5, Fueron codificados de manera inversa.

Como los ítems se respondían en una escala de 5 puntos a nivel ordinal, se utilizó para el análisis factorial exploratorio la matriz de correlación policórica. Se verificó la adecuación de esta matriz para el análisis factorial exploratorio usando la prueba de esfericidad de Bartlett y el índice KMO. Posteriormente, se estableció el número de factores utilizando el análisis paralelo de Horn para datos policóricos con 500 muestras, complementado con el resultado de VSS, MAP de Velicer y BIC. El análisis factorial exploratorio se realizó utilizando extracción de mínimos cuadrados generalizados y rotación geomin oblicua. Se procedió a optimizar la solución eliminando ítems de forma iterativa hasta lograr una estructura simple, en la cual cada ítem tuviere solo en un factor con carga igual o superior a 4., con una diferencia de al menos, 15 con respecto a la segunda carga más fuerte. Considerando que la solución contaba con 6 factores todos relacionados entre sí, se decide explorar una estructura factorial de segundo orden, la que resultó satisfactoria.

En la submuestra confirmatoria, se realizó análisis factorial confirmatorio de 3 modelos anidados usando SEM, en orden de restricción: 1) estructura unidimensional para todos los ítems, 2) estructura jerárquica de 1 factor de segundo orden que comprende los 6 factores de primer orden y, 3) solución de 6 factores de primer orden, correlacionados. Se utilizó estimación WLSMV, que es adecuada para escalas conformadas por ítems a nivel ordinal de menos de 5 puntos. Se evaluó cada modelo usando Chi cuadrado (X2), $X 2$ normalizado (X2/gl), CFI, TLI y RMSEA. Los criterios usados para evaluar adecuadamente el modelo fueron: (a) $x 2 / g \mid<3$, (b) CFI y TLI mayores a 0.9 corresponden a un ajuste aceptable y sobre 0,95 a un buen ajuste, (c) RMSEA con valores menores a 0.08 indican un ajuste aceptable y menores a 0.06 un buen ajuste (Hu y Bentler, 1999). Para probar la diferencia entre el modelo jerárquico y de 6 factores correlacionados, se estimaron ambos modelos con estimación PML y que utilizó una prueba de diferencia de $X^{2}$ escalados, que resulta apta para ítems de nivel ordinal (Katsikatsou y Moustaki, 2016).

Se analizó la confiabilidad de las escalas en las 2 submuestras usando alfa ordinal, basado en la correlación policórica de los ítems, y omega total, para los factores de primer orden y la suma del total de ítems. Como este último indicador considera tanto la varianza del factor general como de los factores de primer orden, se calculó omega jerárquico para el factor general de segundo orden, el cual solo considera la varianza atribuible al factor de segundo orden, excluyendo la de atribuible a los factores de primer orden.

\section{RESULTADOS}

Para dar respuesta al objetivo de diseñar y validar el cuestionario de expectativas hacia la educación virtual en estudiantes universitarios durante la pandemia por COVID-19, en la tabla 2 se presentan los descriptivos de la escala de expectativas de los y las estudiantes sobre la educación virtual. Se puede observar que 3 ítems presentan asimetrías o curtosis fuera del rango $[-1,+1]$, lo que en el caso del ítem 12 (creo que mis oportunidades de aprender en esta modalidad virtual serán (menor/igual/mayores) que en la clase tradicional / presencial), se explica por qué la media, $M=1.61$, es la menor de todos los ítems se encuentra cercana al mínimo de la escala, siendo la asimetría y curtosis positiva. En cambio, los ítems 34 (creo que seré capaz de ejecutar procesos administrativos de mi carrera de manera online inscribir asignaturas, obtener claves, extraer informes, etc.) y el ítem 8 (creo que la diversidad de recursos y actividades es importante para un mejor aprendizaje), son los ítems con medias más altas, presentando asimetría negativa y también curtosis altas.

\section{Análisis factorial exploratorio}

Cómo los ítems se miden a nivel ordinal en 5 niveles, se utiliza la matriz de correlaciones policóricas, El índice $\mathrm{KMO}=.96$ y la prueba de esfericidad de Bartlett, $\mathrm{X}^{2}(666)=105647,663, p<0,001$, muestran que la matriz de correlaciones policórica es apta para el análisis factorial. El análisis paralelo de Horn usando 500 remuestras con matriz de correlaciones polícóricas indicó que la solución factorial apropiada es de 9 factores. Por su parte, el criterio VSS indica una solución de 1 factor, Map de Velicer una solución de 5 y BIC una solución de 8. Considerando la gran variedad de número de factores, se puede asumir que es posible la existencia de una estructura jerárquica, de 1 a 5 factores, que comprende a 8 o 9 factores primarios. Por tanto, se probó de forma inicial una solución de 9 factores. 
Tabla 2: Descriptivos de escala CEEVESE

\begin{tabular}{|c|c|c|c|c|c|c|}
\hline Ítem & Dimensión & Texto & M & DE & Asimetría & Curtosis \\
\hline 1 & \multirow{4}{*}{$\begin{array}{l}\text { Compromiso } \\
\text { institucional }\end{array}$} & Percibo que mi Universidad está comprometida con la educación virtual & 3.55 & 0.98 & -0.70 & 0.16 \\
\hline 2 & & $\begin{array}{l}\text { Creo que en mi Universidad los directivos (directores de departamento / jefes de carrera) } \\
\text { proveerán apoyo a los profesores para un buen proceso formativo en la virtualidad }\end{array}$ & 3.46 & 0.98 & -0.54 & -0.04 \\
\hline 3 & & Considero que mi Universidad proveerá apoyo en el ámbito tecnológico & 3.58 & 0.97 & -0.68 & 0.21 \\
\hline 4 & & Siento que mi aprendizaje en el aula virtual va a ser autodidacta & 2.26 & 1.09 & 0.72 & -0.08 \\
\hline 5 & \multirow{8}{*}{$\begin{array}{l}\text { Calidad en los } \\
\text { procesos de } \\
\text { Aprendizaje }\end{array}$} & $\begin{array}{l}\text { Las plataformas disponibles (CANVAS, INFODA y Campus Virtual) me ayudarán a } \\
\text { alcanzar los resultados de aprendizaje de mis asignaturas }\end{array}$ & 2.91 & 1.04 & -0.19 & -0.69 \\
\hline 6 & & $\begin{array}{l}\text { Creo que los recursos y actividades virtuales de las asignaturas provistas por los } \\
\text { profesores (ppt, infografías, videos, simuladores u otro) ayudarán a mi aprendizaje }\end{array}$ & 3.53 & 0.96 & -0.76 & 0.27 \\
\hline 7 & & $\begin{array}{l}\text { Creo que las actividades en línea provistos por los profesores (foros, evaluación por pares, } \\
\text { video conferencias y otras) ayudarán a mi aprendizaje }\end{array}$ & 3.36 & 1.01 & -0.56 & -0.24 \\
\hline 8 & & Creo que la diversidad de recursos y actividades es importante para un mejor aprendizaje & 4.04 & 0.87 & -1.05 & 1.59 \\
\hline 9 & & Creo que las clases virtuales ayudarán a mi aprendizaje & 2.79 & 1.12 & -0.04 & -0.85 \\
\hline 10 & & $\begin{array}{l}\text { Considero que las tareas evaluativas que se llevarán a cabo en el aula virtual contribuirán } \\
\text { a mi aprendizaje }\end{array}$ & 2.96 & 1.06 & -0.25 & -0.62 \\
\hline 11 & & $\begin{array}{l}\text { Creo que mi rendimiento académico (nota), en esta modalidad virtual, será } \\
\text { (peor/igual/mejor) que en la clase tradicional / presencial }\end{array}$ & 2.13 & 1.37 & 0.81 & -0.54 \\
\hline 12 & & $\begin{array}{l}\text { Creo que mis oportunidades de aprender en esta modalidad virtual serán } \\
\text { (menor/igual/mejor) que en la clase tradicional / presencial }\end{array}$ & 1.61 & 1.14 & 1.73 & 1.94 \\
\hline 13 & \multirow{7}{*}{$\begin{array}{l}\text { Calidad en los } \\
\text { procesos de } \\
\text { Enseñanza }\end{array}$} & Creo que mis cursos se desarrollarán de acuerdo a lo planificado & 2.87 & 1.06 & -0.10 & -0.81 \\
\hline 14 & & $\begin{array}{l}\text { Creo que la información sobre cómo se desarrollará las asignaturas en línea será } \\
\text { entregada de forma clara, }\end{array}$ & 3.12 & 1.06 & -0.34 & -0.71 \\
\hline 15 & & $\begin{array}{l}\text { Creo que la información sobre cómo se desarrollarán las asignaturas en línea será } \\
\text { entregada a tiempo, }\end{array}$ & 3.13 & 1.04 & -0.35 & -0.64 \\
\hline 16 & & Creo que los docentes serán responsables en sus horarios de clases en línea & 3.61 & 1.06 & -0.76 & 0.08 \\
\hline 17 & & Creo que los docentes serán responsables con sus horarios de atención en línea & 3.57 & 0.98 & -0.66 & 0.16 \\
\hline 18 & & Creo que las evaluaciones a realizar en línea tendrán la exigencia adecuada & 3.08 & 1.01 & -0.30 & -0.40 \\
\hline 19 & & Creo que las evaluaciones a realizar en línea tendrán el tiempo necesario & 2.95 & 1.08 & -0.21 & -0.70 \\
\hline 20 & \multirow{7}{*}{$\begin{array}{l}\text { Calidad en la } \\
\text { relación con } \\
\text { pares }\end{array}$} & $\begin{array}{l}\text { Creo que usaré los medios de comunicación (foro, chat, correo, video conferencia u otro) } \\
\text { del aula virtual para comunicarme con mis compañeros }\end{array}$ & 3.32 & 1.15 & -0.47 & -0.62 \\
\hline 21 & & $\begin{array}{l}\text { Creo que el aula virtual me dará más oportunidades de relacionarme con compañeros/as } \\
\text { que de forma presencial }\end{array}$ & 1.93 & 1.03 & 0.94 & 0.17 \\
\hline 22 & & $\begin{array}{l}\text { Creo que el usar los medios de comunicación virtual me ayudará a establecer relaciones } \\
\text { que no hubiera conseguido de manera presencial }\end{array}$ & 1.98 & 1.04 & 0.82 & -0.13 \\
\hline 23 & & $\begin{array}{l}\text { Creo que los recursos y actividades en línea permitirán trabajar de manera más } \\
\text { cooperativa con mis compañeros }\end{array}$ & 2.36 & 1.10 & 0.29 & -0.88 \\
\hline 24 & & $\begin{array}{l}\text { Creo que trabajar con mis compañeros en línea me ayudará a obtener mejores } \\
\text { aprendizajes }\end{array}$ & 2.36 & 1.10 & 0.31 & -0.79 \\
\hline 25 & & $\begin{array}{l}\text { Creo que la relación con mis compañeros en la virtualidad será (peor/ igual / mejor) que en } \\
\text { la tradicional / presencial }\end{array}$ & 2.05 & 1.14 & 0.53 & -0.70 \\
\hline 26 & & $\begin{array}{l}\text { Creo que el trabajo grupal en la virtualidad será (peor / igual / mejor) que en el tradicional / } \\
\text { presencial }\end{array}$ & 1.85 & 1.16 & 0.99 & -0.02 \\
\hline 27 & \multirow{2}{*}{$\begin{array}{l}\text { Calidad en la } \\
\text { relación con } \\
\text { los docentes }\end{array}$} & $\begin{array}{l}\text { Creo que los docentes serán capaces de utilizar las herramientas del aula virt } \\
\text { mejorar mi aprendizaje }\end{array}$ & 3.09 & 1.01 & -0.33 & -0.50 \\
\hline 28 & & $\begin{array}{l}\text { Creo que los docentes estarán atentos a cómo se desarrolla mi aprendizaje en el aula } \\
\text { virtual }\end{array}$ & 3.05 & 1.08 & -0.25 & -0.69 \\
\hline 29 & \multirow{2}{*}{$\begin{array}{l}\text { Calidad en la } \\
\text { relación con } \\
\text { los docentes }\end{array}$} & $\begin{array}{l}\text { Creo que los docentes estarán disponibles en línea para responder a mis inquietudes } \\
\text { académicas }\end{array}$ & 3.52 & 0.97 & -0.70 & 0.28 \\
\hline 30 & & $\begin{array}{l}\text { Creo que mi relación con los docentes en la virtualidad será (peor / igual / mejor) que en la } \\
\text { tradicional / presencial }\end{array}$ & 2.29 & 1.20 & 0.33 & -0.67 \\
\hline 31 & \multirow{7}{*}{$\begin{array}{l}\text { Autoeficacia } \\
\text { percibida para } \\
\text { la educación } \\
\text { virtual }\end{array}$} & Creo que seré capaz de usar los recursos del aula virtual para aprender & 3.62 & 0.97 & -0.84 & 0.45 \\
\hline 32 & & $\begin{array}{l}\text { Creo que seré capaz de usar los recursos del aula virtual más allá de lo que pide el } \\
\text { docente }\end{array}$ & 3.22 & 1.02 & -0.28 & -0.34 \\
\hline 33 & & $\begin{array}{l}\text { Creo que seré capaz de trabajar en equipo con mis compañeros utilizando las } \\
\text { herramientas virtuales }\end{array}$ & 3.25 & 1.05 & -0.52 & -0.40 \\
\hline 34 & & $\begin{array}{l}\text { Creo que seré capaz de ejecutar procesos administrativos de mi carrera de manera online } \\
\text { (inscribir asignaturas, obtener claves, extraer informes, etc,) }\end{array}$ & 3.73 & 0.95 & -1.01 & 1.07 \\
\hline 35 & & $\begin{array}{l}\text { Considero que seré capaz de cumplir con todas las actividades de aprendizaje del aula } \\
\text { virtual }\end{array}$ & 3.40 & 1.05 & -0.58 & -0.26 \\
\hline 36 & & $\begin{array}{l}\text { Creo que seré capaz de organizar mi tiempo de manera efectiva para responder a las } \\
\text { necesidades de la educación en línea y de los otros ámbitos de mi vida (recreación, } \\
\text { familia, intereses, etc,), }\end{array}$ & 3.02 & 1.20 & -0.19 & -0.97 \\
\hline 37 & & $\begin{array}{l}\text { Creo que mi rendimiento este semestre va a ser (muy malo / malo / regula / bueno / muy } \\
\text { bueno) }\end{array}$ & 2.98 & 0.92 & -0.21 & 0.01 \\
\hline
\end{tabular}


La solución inicial, que explica un $63 \%$, no es adecuada, ya que los ítems $27,4,30$ y 20 no muestran cargas superiores a 0,4 en ningún factor y el ítem 33 presenta una carga cruzada y el factor 9 no presenta ítems suficientes. Por tanto, se procedió a mejorar de forma iterativa la solución de acuerdo con lo presentado en la tabla 3, tratando de lograr una solución simple, con cargas superiores a 0,4 en cada factor y sin cargas cruzadas. Esta solución se logró tras 15 iteraciones, manteniendo 27 ítems y 5 factores. Esta información es descrita en la tabla 3.

Tabla 3: Proceso iterativo de mejora de la solución factorial del CEEVESE

\begin{tabular}{|c|c|c|c|c|c|}
\hline$N,{ }^{\circ}$ solución & Ítems & Factores & $\begin{array}{l}\text { Varianza } \\
\text { explicada }\end{array}$ & Problemas detectados & Acción \\
\hline 1 & 37 & 9 & $63 \%$ & $\begin{array}{l}\text { Sin carga suficiente: } 30,27,4,20 \\
\text { Carga cruzada: } 33 \\
\text { Factor } 9 \text { sin cargas suficientes }\end{array}$ & Se elimina factor 9 \\
\hline 2 & 37 & 8 & $61 \%$ & $\begin{array}{l}\text { Sin carga suficiente: } 27,30,4,15,20,14,13 \\
\text { Carga cruzada: } 33 \\
\text { Factor } 8 \text { sin cargas suficientes }\end{array}$ & Se elimina factor 8 \\
\hline 3 & 37 & 7 & $60 \%$ & $\begin{array}{l}\text { Sin carga suficiente: } 19,30,18,14,4,20,13 \\
\text { Carga cruzada: } 33 \\
\text { Factor } 7 \text { sin cargas suficientes }\end{array}$ & Se elimina factor 7 \\
\hline 4 & 37 & 6 & $58 \%$ & $\begin{array}{l}\text { Sin carga suficiente: } 4,1,2,20,30,19,18,3 \\
\text { Carga cruzada: } 33 \\
\text { Ítem en factor único: } 13\end{array}$ & Se elimina ítem 33 \\
\hline 5 & 36 & 6 & $58 \%$ & $\begin{array}{l}\text { Sin carga suficiente: } 4,19,27,30,20 \\
\text { Carga cruzada: } 3\end{array}$ & Se elimina ítem 3 \\
\hline 6 & 35 & 6 & $59 \%$ & $\begin{array}{l}\text { Sin carga suficiente: } 4,30,20 \\
\text { Carga cruzada: } 17\end{array}$ & Se elimina ítem 20 \\
\hline 7 & 34 & 6 & $59 \%$ & $\begin{array}{l}\text { Sin carga suficiente: } 4,30 \\
\text { Carga cruzada: } 17\end{array}$ & Se elimina ítem 30 \\
\hline 8 & 33 & 6 & $60 \%$ & $\begin{array}{l}\text { Sin carga suficiente: } 4 \\
\text { Carga cruzada: } 17,16\end{array}$ & Se elimina 17 \\
\hline 9 & 32 & 6 & $59 \%$ & $\begin{array}{l}\text { Sin carga suficiente: } 4,19 \\
\text { Carga cruzada: } 18,37\end{array}$ & Se elimina 37 \\
\hline 10 & 31 & 6 & $59 \%$ & Sin carga suficiente: 4 & Se elimina 4 \\
\hline 11 & 30 & 6 & $61 \%$ & Sin carga suficiente: 13,8 & Se elimina 8 \\
\hline 12 & 29 & 6 & $62 \%$ & Sin carga suficiente: 13 & Se elimina 13 \\
\hline 13 & 28 & 6 & $62 \%$ & Sin problemas & \\
\hline
\end{tabular}

En la tabla 4 se presenta la solución factorial final de 28 ítems y 6 factores, que explica un $62 \%$ de la varianza. El factor 1, que denominaremos Relación con compañeros, comprende los ítems 21,22, 23,24, 25 y 26, y da cuenta de la posibilidad que tienen los y las estudiantes de relacionarse con las y los compañeros en línea. El factor 2, de Aprendizaje en línea, reúne a los ítems 5, 6, 7, 9 y 10, que dan cuenta de la expectativa que los recursos en línea ayudarán al aprendizaje. El factor 3 , que denominaremos Docencia en Línea, comprende los ítems $1,2,14,15,16,27,28$ y 29, que dan cuenta de la expectativa que la universidad está comprometida con la enseñanza en línea, que los y las docentes realizarán sus cursos de acuerdo con lo planificado, estarán disponibles y atentos a los procesos de aprendizaje y que serán capaces de utilizar las herramientas del aula virtual para enseñar. El factor 4, de Autoeficacia para el aprendizaje en línea, comprende los ítems 31, 32, 34, 35,36 , los cuales indican la capacidad para realizar acciones que promuevan el aprendizaje en línea. El factor 5, de Evaluación en Línea, considera los ítems 18 y 19, que dan cuenta de las expectativas sobre la exigencia y el tiempo de las evaluaciones en línea. Finalmente, el factor 6 que denominaremos Comparación con la educación presencial, comprende los ítems 11 y 12, que comparan el rendimiento y aprendizaje en línea con el presencial. Al analizar las correlaciones entre los factores, podemos ver que la media es de $r=0,50$, estando el rango de correlaciones media por factor entre 0,34 para el factor 6 y 0,55 para el factor 2 . Por lo tanto, resulta adecuado probar la pertinencia de un modelo factorial de segundo orden con un factor general. Para ello, se ajusta un análisis factorial exploratorio de un factor de segundo orden, que explica un $49 \%$ de la varianza de los factores de primer orden. Se puede ver que todas las cargas factoriales superan el 0,4 y las cargas de las dimensiones Aprendizaje en línea (F2), Docencia en línea (F3), Autoeficacia para el aprendizaje en línea (F4) y Evaluación en línea (F5) superan el 0,7. 
Tabla 4: Solución factorial exploratoria del CEEVESE

\begin{tabular}{|c|c|c|c|c|c|c|}
\hline Ítem & $\begin{array}{l}\text { Relación con } \\
\text { compañeros (F1) }\end{array}$ & $\begin{array}{l}\text { Aprendizaje en } \\
\text { línea (F2) }\end{array}$ & $\begin{array}{l}\text { Docencia en } \\
\text { línea (F3) }\end{array}$ & $\begin{array}{c}\text { Autoeficacia para el } \\
\text { aprendizaje en línea (F4) }\end{array}$ & $\begin{array}{l}\text { Evaluación en } \\
\text { línea (F5) }\end{array}$ & $\begin{array}{c}\text { Comparación con la } \\
\text { educación presencial (F6) }\end{array}$ \\
\hline Ítem 22 & 0.86 & 0.01 & 0.00 & -0.02 & 0.01 & -0.01 \\
\hline Ítem 21 & 0.84 & 0.03 & -0.02 & 0.01 & 0.01 & -0.07 \\
\hline Ítem 23 & 0.77 & 0.07 & 0.04 & 0.00 & 0.02 & 0.02 \\
\hline Ítem 25 & 0.67 & -0.16 & 0.03 & 0.02 & -0.04 & 0.21 \\
\hline Ítem 24 & 0.59 & 0.16 & -0.02 & 0.07 & 0.06 & 0.02 \\
\hline Ítem 26 & 0.52 & -0.03 & 0.03 & -0.02 & 0.02 & 0.37 \\
\hline Ítem 09 & 0.07 & 0.77 & -0.02 & 0.02 & 0.04 & 0.09 \\
\hline Ítem 06 & -0.06 & 0.76 & 0.14 & 0.00 & 0.01 & 0.01 \\
\hline Ítem 07 & -0.01 & 0.76 & 0.08 & 0.05 & 0.03 & -0.04 \\
\hline Ítem 05 & 0.01 & 0.71 & 0.09 & 0.02 & 0.04 & 0.05 \\
\hline Ítem 10 & 0.08 & 0.61 & -0.05 & 0.06 & 0.22 & 0.02 \\
\hline Ítem 16 & -0.06 & -0.04 & 0.72 & -0.02 & 0.06 & 0.07 \\
\hline Ítem 29 & 0.01 & -0.04 & 0.68 & 0.11 & 0.01 & 0.04 \\
\hline Ítem 27 & 0.06 & 0.18 & 0.63 & 0.03 & 0.03 & -0.03 \\
\hline Ítem 28 & 0.08 & 0.02 & 0.61 & 0.08 & 0.05 & -0.02 \\
\hline Ítem 15 & -0.01 & 0.04 & 0.60 & 0.02 & 0.19 & 0.04 \\
\hline Ítem 02 & 0.04 & 0.25 & 0.57 & -0.01 & -0.02 & -0.09 \\
\hline Ítem 14 & 0.03 & 0.08 & 0.56 & 0.04 & 0.15 & 0.03 \\
\hline Ítem 01 & -0.02 & 0.37 & 0.53 & -0.03 & -0.06 & 0.00 \\
\hline Ítem 32 & 0.07 & 0.01 & -0.04 & 0.81 & -0.01 & -0.04 \\
\hline Ítem 35 & -0.01 & -0.04 & 0.05 & 0.80 & 0.09 & 0.00 \\
\hline Ítem 31 & 0.00 & 0.16 & 0.04 & 0.75 & -0.07 & 0.04 \\
\hline Ítem 34 & -0.03 & 0.01 & 0.13 & 0.60 & -0.01 & 0.03 \\
\hline Ítem 36 & 0.04 & 0.03 & 0.01 & 0.55 & 0.16 & 0.01 \\
\hline Ítem 18 & 0.03 & 0.06 & 0.09 & -0.03 & 0.72 & 0.02 \\
\hline Ítem 19 & 0.01 & -0.01 & 0.07 & 0.06 & 0.71 & -0.01 \\
\hline Ítem 12 & 0.09 & 0.37 & 0.06 & 0.01 & -0.04 & 0.58 \\
\hline Ítem 11 & -0.02 & 0.15 & -0.12 & 0.25 & 0.11 & 0.51 \\
\hline
\end{tabular}

Al analizar los residuos de la solución factorial exploratoria, se observa dos correlaciones residuales de relevancia, que se explican en función de los factores que se eliminan por sus bajas cargas factoriales en el proceso iterativo de optimización. La primera correlación residual, de $r=0,15$, se produce entre los ítems 1 , (Percibo que mi Universidad está comprometida con la educación virtual), y el ítem 2, (Creo que en mi Universidad los directivos (directores de departamento / jefes de carrera) proveerán apoyo a los profesores para un buen proceso formativo en la virtualidad). Estos ítems en la solución inicial forman uno de los 9 factores iniciales, compuesto por los ítems 1,2 y 3 , siendo absorbidos por el factor 3 al eliminarse el ítem 3. En términos teóricos, presentan una relación de causalidad implícita - si la universidad está comprometida, se asume que proveerá de apoyo a los docentes. La segunda correlación residual, de $r=0,17$, corresponde a la presente entre los ítems 14, (Creo que la información sobre cómo se desarrollará las asignaturas en línea será entregada de forma clara) y el ítem 15 (Creo que la información sobre cómo se desarrollarán las asignaturas en línea será entregada a tiempo). En la solución inicial, uno de los 9 factores está conformado por los ítems 13,14 y 15, el cual termina siendo absorbido en el factor 3 al eliminar el ítem 13; si analizamos presentan tanto una redacción muy similar, así como refieren al mismo fenómeno, que es la entrega de información sobre la asignatura. Considerando que estas dos relaciones reflejan relación fuerte entre ítems, que luego fueron consideradas dentro del factor 3 , se incluirán como correlaciones entre residuos en los modelos a estudiar en el análisis confirmatorio descritos en la tabla 5. 


\section{Análisis factorial confirmatorio}

En la tabla 6 se describen los resultados del análisis de 3 modelos anidados: Modelo unidimensional (M1), el modelo jerárquico con un factor general de segundo orden y 6 factores relacionados (M2) y el modelo de 6 factores relacionados (M3). Por el gran tamaño de la muestra, se puede ver que el criterio $\mathrm{X}^{2} / \mathrm{gl}$ supera ampliamente el valor 3 , por lo que no resulta un buen indicador de ajuste. Usando el resto de los indicadores, se puede ver que el modelo M1, unidimensional, es claramente inaceptable, porque, CFI y $T L I<0,90$, así como RMSEA $>0,8$. Si bien la prueba de diferencia de $X^{2}$ escalados usando estimación PLM, muestra una diferencia estadísticamente significativa entre ambos modelos, $X^{2}(7,08)=257,42, p<0,001$, lo que nos indica que el modelo M3 se ajusta mejor a los datos que el modelo M2, ambos modelos presentan buenos indicadores de ajustes relativos. En el caso de CFI y TLI, para ambos modelos son mayores a 0,95 y SRMR, es menor a 0,05 también en ambos modelos. En el caso de RMSEA, ambos modelos presentan un desempeño de adecuado a bueno, presentando el modelo M2 jerárquico RMSEA $=0,062$ y el modelo M3 de 6 factores relacionados RMSEA $=0,060$. Aunque los modelos M2 y M3 son aceptables desde el punto de vista de los indicadores de ajuste relativo.

Tabla 5: Correlaciones entre factores y carga factorial en factor de segundo orden del CEEVESE

\begin{tabular}{|l|c|c|c|c|c|c|c|}
\hline Factor & $\begin{array}{c}\text { Relación con } \\
\text { compañeros } \\
\text { (F1) }\end{array}$ & $\begin{array}{c}\text { Aprendizaje } \\
\text { en línea (F2) }\end{array}$ & $\begin{array}{c}\text { Docencia en } \\
\text { línea (F3) }\end{array}$ & $\begin{array}{c}\text { Autoeficacia para el } \\
\text { aprendizaje en línea } \\
\text { (F4) }\end{array}$ & $\begin{array}{c}\text { Evaluación en } \\
\text { línea (F5) }\end{array}$ & $\begin{array}{c}\text { Comparación con la } \\
\text { educación presencial } \\
\text { (F6) }\end{array}$ & $\begin{array}{c}\text { Carga } \\
\text { factorial }\end{array}$ \\
\hline $\begin{array}{l}\text { Relación con } \\
\text { compañeros (F1) }\end{array}$ & 1 & 0.5 & 0.37 & 0.42 & 0.43 & 0.34 \\
\hline $\begin{array}{l}\text { Aprendizaje en línea } \\
\text { (F2) }\end{array}$ & & 1 & 0.6 & 0.69 & 0.59 & 0.37 \\
\hline $\begin{array}{l}\text { Docencia en línea } \\
\text { (F3) }\end{array}$ & & & 1 & 0.55 & 0.59 & 0.25 \\
\hline $\begin{array}{l}\text { Autoeficacia para el } \\
\text { aprendizaje en línea } \\
\text { (F4) }\end{array}$ & & & & 1 & 0.55 & 0.84 \\
\hline $\begin{array}{l}\text { Evaluación en línea } \\
\text { (F5) }\end{array}$ & & & & & 1 & 0.45 \\
\hline $\begin{array}{l}\text { Comparación con la } \\
\text { educación presencial } \\
\text { (F6) }\end{array}$ & & & & & & 0.28 \\
\hline
\end{tabular}

Al analizar los residuos promedios por ítem para los modelos M2 y M3, estos son muy similares, excepto por residuos promedios más altos en los ítems 11 y 12, lo que se puede explicar por la presencia de correlaciones de los residuos de estos ítems con todos los factores excepto el 4, que no son explicadas por el factor general (tabla 4). En términos de los índices de modificación, el principal es una correlación entre residuos positiva entre los ítems 21 y 22 tanto en el modelo jerárquico $(\mathrm{Ml}=708,8)$ como en el de 6 factores relacionados $(\mathrm{Ml}=709,9)$.

Tabla 6: Análisis factorial confirmatorio del CEEVESE

\begin{tabular}{|l|c|c|c|c|c|c|}
\hline \multicolumn{1}{|c|}{ Modelo } & \multicolumn{1}{|c|}{$\mathrm{X}^{2}$} & $\mathrm{X}^{2} / \mathrm{gl}$ & CFI & TLI & SRMR & RMSEA \\
\hline M1: Unidimensional & $\mathrm{X}^{2}(350)=27021.73 . \mathrm{p}<0.001$ & 77.205 & .791 & .774 & .096 & $0.136[0.134 .0 .137] . \mathrm{p}<0.001$ \\
\hline M2: Jerárquico & $\mathrm{X}^{2}(344)=5774.07 . \mathrm{p}<0.001$ & 16.785 & .957 & .953 & .047 & $0.062[0.060 .0 .063] . \mathrm{p}<0.001$ \\
\hline M3: 6 Factores relacionados & $\mathrm{X}^{2}(335)=5354.88 . \mathrm{p}<0.001$ & 15.985 & .961 & .956 & .041 & $0.060[0.059 .0 .062] . \mathrm{p}<0.001$ \\
\hline
\end{tabular}

En la Figura 1 y 2 se observa que las cargas factoriales son superiores en todos los ítems a 0,6. Las correlaciones de $\mathrm{F} 1$ con los otros 5 factores se encuentran en el rango 0,50 a 0,59, lo que explica su carga factorial menor con el factor general. Las dimensiones fueron identificadas de la siguiente manera: F1: Relación con compañeros, F2: Aprendizaje en línea, F3: Docencia en línea, F4: Autoeficacia para el aprendizaje en línea, F5: Evaluación en línea, F6: Comparación con la educación presencial. En este caso, se presenta la distribución de primero el modelo M2 jerárquico y el modelo M3 de 6 factores correlacionados. 

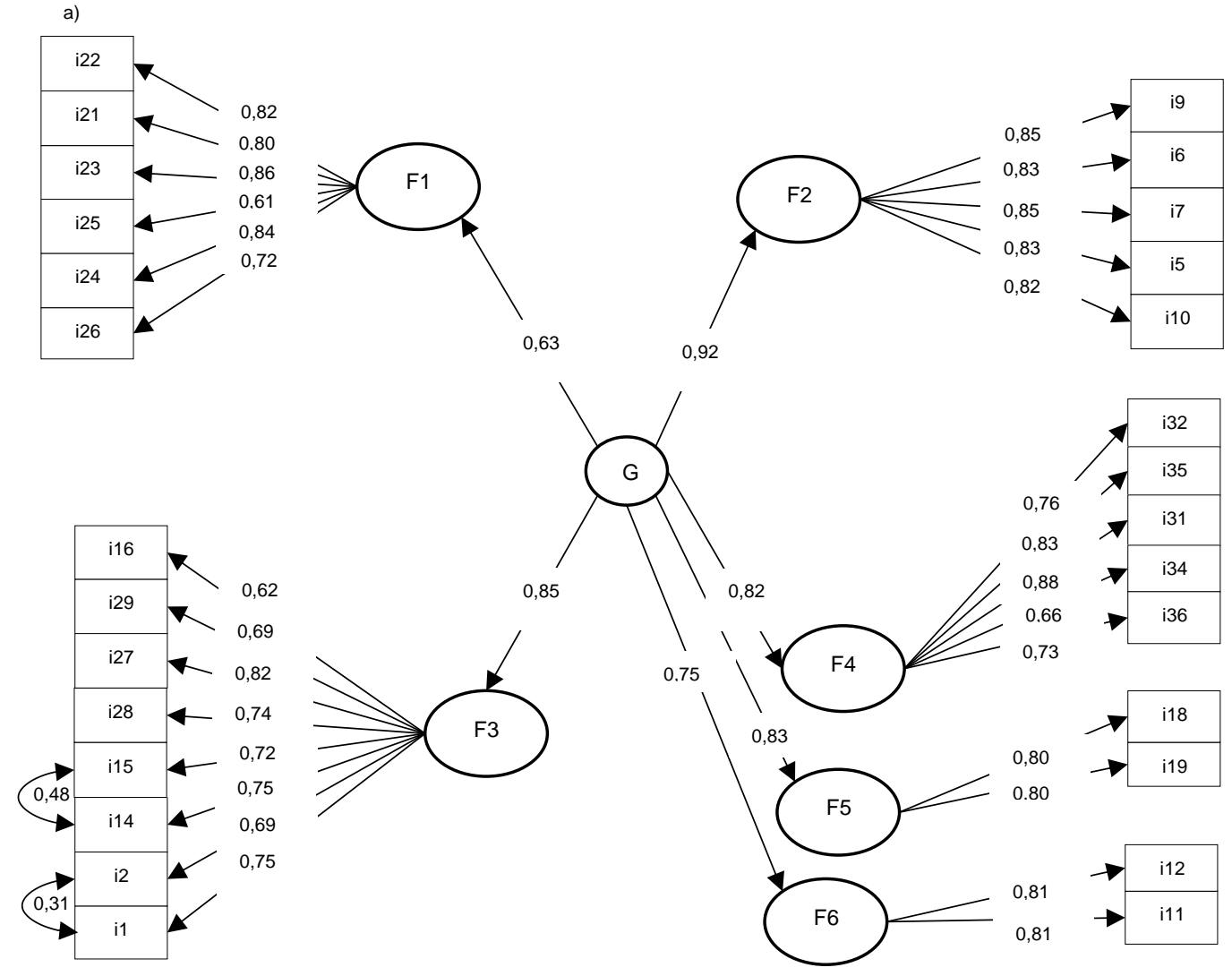

Fig. 1: Modelo M2 jerárquico del CEEVESE

b)
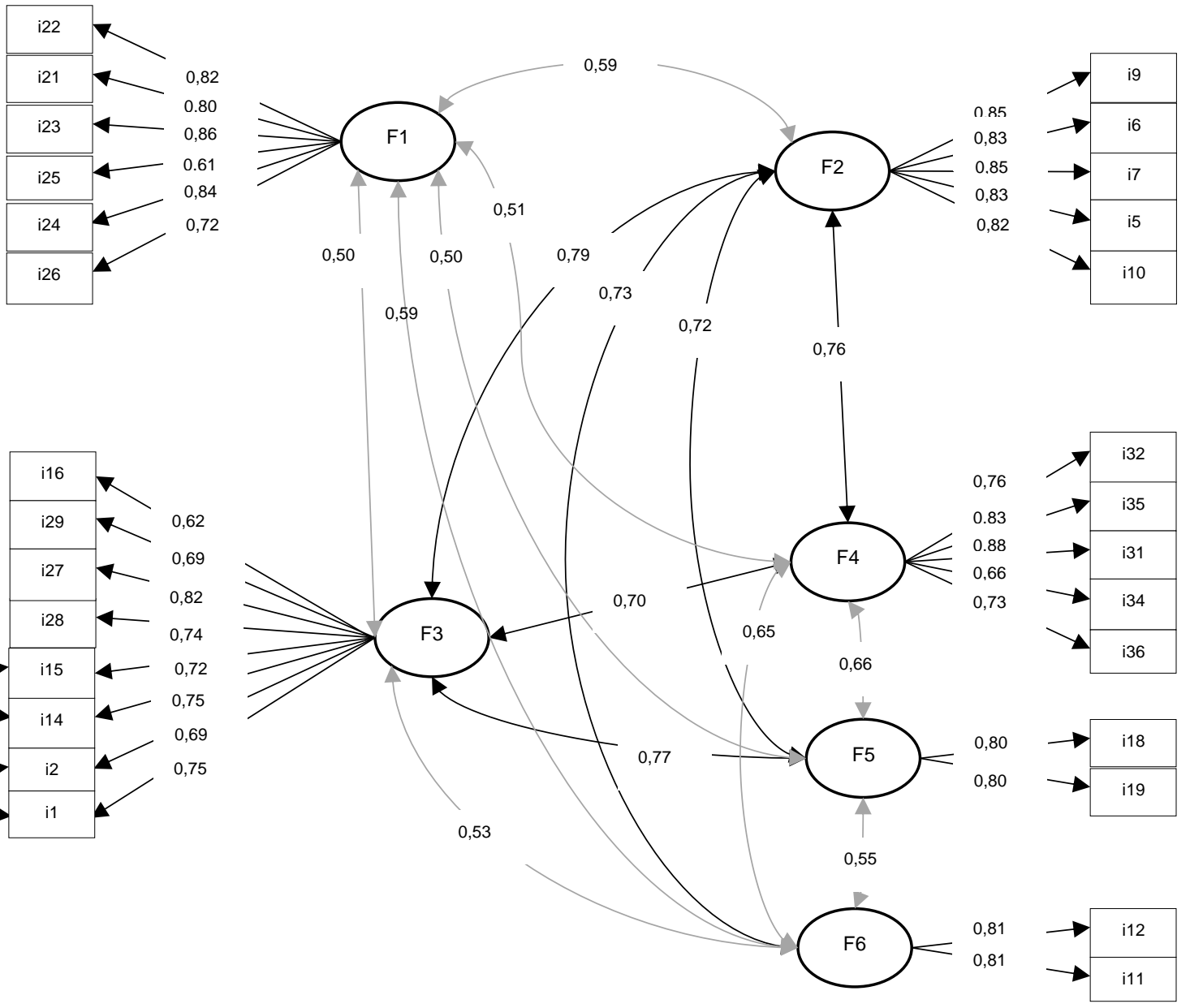

Fig. 2: Modelo M3 de 6 factores relacionados del CEEVESE 


\section{Análisis de confiabilidad}

En la tabla 7 se presentan los indicadores de consistencia interna alfa ordinal $(\alpha 0)$ y omega total ( $\omega t)$ para todas las escalas de la solución del modelo M3 de 6 factores relacionados. Como se puede ver, todas las escalas excepto en la submuestra en el factor F6, presentan confiabilidades sobre 0,70 que es el mínimo aceptable. Para la suma total de ítems, el $\alpha$ o para ambas escalas es 0,95 , lo que es alto, pero considera tanto la varianza atribuible al factor general como a los factores específicos; el omega total, que se basa en la varianza común a todos los ítems, presenta un valor de 0,87 en ambas submuestras. Si analizamos el omega jerárquico, que considera solo la varianza atribuible el factor general del modelo $\mathrm{M} 2$, este es de $\omega \mathrm{t}=0,88$ para la submuestra 1 y $\omega t=0,87$ para la muestra 2 , muy similar a la omega total calculado para el total de ítems en el modelo M1 de 6 factores correlacionados.

Tabla 7: Indicadores de consistencia interna para los factores en las submuestras 1 y 2 de la solución de 6 factores relacionados del CEEVESE

\begin{tabular}{|l|c|c|c|c|}
\hline & \multicolumn{2}{|l|}{ Submuestra 1 } & \multicolumn{2}{l|}{ Submuestra 2 } \\
\hline Factor & $\alpha \circ$ & $\omega t$ & $\alpha \circ$ & $\omega t$ \\
\hline Relación con compañeros (F1) & 0,894 & 0,881 & 0,892 & 0,881 \\
\hline Aprendizaje en línea (F2) & 0,922 & 0,901 & 0,918 & 0,897 \\
\hline Docencia en línea (F3) & 0,907 & 0,731 & 0,900 & 0,734 \\
\hline Autoeficacia para el aprendizaje en línea (F4) & 0,882 & 0,858 & 0,875 & 0,851 \\
\hline Evaluación en línea (F5) & 0,787 & 0,743 & 0,786 & 0,743 \\
\hline Comparación con la educación presencial (F6) & 0,779 & 0,657 & 0,793 & 0,671 \\
\hline Total & 0,954 & 0,873 & 0,952 & 0,874 \\
\hline
\end{tabular}

\section{Distribución de las escalas}

En la tabla 8 se pueden observar los descriptivos para las 6 escalas resultantes del análisis factorial, así como la puntuación total. Se puede ver que las escalas con menor puntuación son $\mathrm{F6}(\mathrm{M}=1,87)$, la comparación con la educación presencial, y $\mathrm{F} 1(\mathrm{M}=2,09)$, relación con compañeros. Por otra parte, las escalas con mayor puntuación son F4 $(M=3,38)$, Autoeficacia para el aprendizaje en línea y F3 ( $M=3,31)$, Docencia en línea. En la puntuación total, se encuentran diferencias significativas por cohorte, $X^{2}(6)=90.56, p<0.001$, facultad $X^{2}(22)=365.38, p<0.001$, experiencia previa en aula virtual, $X^{2}(6)=60.722, p<0.001$, evaluación de competencia $X^{2}(3)=1009.1, p<0.001$, y evaluación de la calidad de la experiencia de aprendizaje en aula virtual, $X^{2}(4)=3878, p<0.001$, pero no así por sexo, $p=0.2175$. Al analizar las puntuaciones por cohorte se distingue un grupo con mayores puntuaciones que comprende las cohortes del 2016 hacia atrás $(M=2.99)$ y el grupo de menor expectativas del año $2019(\mathrm{M}=2.80)$. Con respecto a las facultades, se distingue claramente un menor nivel de expectativas de Humanidades y Arte $(M=2.59)$, vs un mayor nivel de expectativas en Ingeniería $(\mathrm{M}=3.05)$. Con respecto a la experiencia previa, los estudiantes con experiencias previas en secundaria o educación superior $(\mathrm{M}=3.10)$, presentan mayores expectativas que aquellos sin experiencia $(\mathrm{M}=2.88)$ o sólo con experiencia el $2019(\mathrm{M}=2.95)$. La evaluación de la habilidad afecta también a las expectativas, siendo mayores en las personas que se consideran avanzadas $(M=3.29)$, vs las personas que se consideran de habilidad media $(M=3.08)$, básica $(M=2.76)$ o nula $(M=2.19)$. Finalmente, las expectativas también se ven influenciadas por la calidad de la experiencia previa, siendo mayor en quienes presentaron muy buenas experiencias de aprendizaje $(M=4.06)$ vs aquellas que la consideraron muy malas $(M=1.96)$.

Tabla 8: Descriptivos para los 6 factores y la suma total del CEEVESE

\begin{tabular}{|c|c|c|c|c|}
\hline Factor & M & DE & Asimetría & Curtosis \\
\hline Relación con compañeros (F1) & 2,09 & 0,82 & 0,60 & $-0,11$ \\
\hline Aprendizaje en línea (F2) & 3,10 & 0,87 & $-0,33$ & $-0,25$ \\
\hline Docencia en línea (F3) & 3,31 & 0,75 & $-0,45$ & 0,19 \\
\hline Autoeficacia para el aprendizaje en línea (F4) & 3,38 & 0,81 & $-0,51$ & 0,38 \\
\hline Evaluación en línea (F5) & 3,01 & 0,94 & $-0,22$ & $-0,31$ \\
\hline Comparación con la educación presencial (F6) & 1,87 & 1,07 & 1,12 & 0,53 \\
\hline Total & 2,90 & 0,65 & $-0,07$ & 0,09 \\
\hline
\end{tabular}




\section{DISCUSIÓN}

La pandemia por COVID-19, ha generado cambios rápidos en la forma como las universidades enseñan hoy en día, por tal motivo, es necesario conocer las expectativas de los y las estudiantes, para así identificar sus preocupaciones, por medio de un instrumento válido y confiable para tal fin, El objetivo de este trabajo fue diseñar y validar el cuestionario de expectativas hacia la educación virtual en estudiantes universitarios. Todo esto, con la finalidad de proporcionar herramientas que permitan la investigación de estos aspectos bajo el escenario producido por la COVID-19 y el desarrollo e implementación de intervenciones que beneficien los procesos de adaptación y de enseñanza-aprendizaje virtual en la Educación Superior.

Luego de revisar las posibles soluciones factoriales del cuestionario CEEVESE, se identificó un ajuste adecuado del modelo. Se estudió la estructura del instrumento usando análisis factorial confirmatorio, encontrándose una solución de 6 factores con buen ajuste, de la cual deriva una solución jerárquica donde todos estos factores dependen de un factor de segundo orden general. La reorganización de los ítems en nuevas dimensiones, semejantes a las planteadas durante la construcción del cuestionario, permite confirmar la evidencia descrita en la literatura sobre la importancia de los elementos asociados a características propias del estudiante, del curso y de las relaciones que establece con los y las integrantes de este, en los procesos de medición de las expectativas de las y los universitarios. Con respecto a la confiabilidad del cuestionario, se identificó índices de consistencia interna adecuados tanto para el factor general como para la mayoría de las dimensiones que constituyen el cuestionario.

La organización de estas seis dimensiones guarda relación con otras investigaciones dentro del contexto de la enseñanza remota de emergencia debido a la pandemia por el COVID-19. Los investigadores han realizado investigaciones con estudiantes universitarios sobre las expectativas frente a la comparación con la educación presencial, a las formas y calidad del aprendizaje, la planificación de los cursos y otros elementos como la interacción con sus compañeros y docentes (Cicha et al., 2021; Imsa-ard, 2020; Suárez Lantarón et al., 2021), adicionalmente una revisión sistemática identificó como elementos relevantes para considerar en la educación virtual, aspectos como la calidad de los materiales de aprendizaje, del entorno virtual, las formas de enseñanza y las experiencias de interacción de los estudiantes con sus pares y profesores (Rodrigues et al., 2019). Estos elementos son coincidentes con las dimensiones generadas durante los procedimientos de validez del cuestionario.

Llama la atención la separación que se produjo en los reactivos asociados a la evaluación en la dimensión de procesos de enseñanza aprendizaje. Los espacios evaluativos serían percibidos por los y las estudiantes como orientados al producto final del ciclo educativo, de carácter social, al estar orientados a constatar y certificar el nivel alcanzado de conocimientos. A este tipo de evaluación se le llama calificación o evaluación sumativa (Aqueveque et al., 2020), en contraste con las llamadas evaluaciones formativa, que se orientan a identificar los cambios que hay que introducir en el proceso de enseñanza para ayudar a los y las estudiantes en su propio proceso de aprendizaje.

Cuando la evaluación es sumativa los docentes pierden oportunidad de proponer tareas complementarias tempranamente y de revisar su enseñanza para tomar decisiones pedagógicas oportunas. Si, además, se estimula un rol activo del estudiante en la evaluación, ésta toma una función reguladora, al promover que el estudiante se haga cargo de su propio proceso de mejora (Hernández et al., 2020). La evaluación formativa es útil para el profesorado en su actuación docente, gratificante para el alumnado en su aprendizaje y orientadora para ambos en sus actuaciones (Hamodi et al., 2015).

La educación en línea ofrece oportunidades mayores y más eficientes para la evaluación formativa (Appiah y Van Tonder, 2018; Wong et al., 2020) que en este caso no se estarían aprovechando. La evaluación por pares (Krouglov, 2021) y el aprendizaje para el dominio (Goksoy, 2018) son estrategias altamente efectivas (McGaghie y Harris, 2018) que podrían estar usándose escasamente. Los hallazgos reportados de que durante ERE los sistemas educativos han tratado de replicar la presencialidad en la virtualidad (Almomani et al., 2021), manteniendo estrategias propias del periodo anterior, podría avalar la mantención de prácticas evaluativas propias de la presencialidad como lo son las pruebas sumativas sincrónicas.

Las expectativas de los estudiantes influyen en sus resultados académicos y en la continuidad en los estudios universitarios (Paechter et al., 2010) fenómeno que también se produce en los contextos de educación virtual. El aprovechamiento de los recursos y actividades para el aprendizaje virtual depende en gran medida de un estudiante que cree en su capacidad de responder a las exigencias del mundo académico (Cai et al., 2017; Regmi y Jones, 2020), por lo que es relevante aumentar este sentido de habilidad de forma que el estudiante despliegue conductas de perseverancia, esfuerzo sostenido y participación en los espacios educativos que no serían posibles si la motivación del estudiante se ve afectada por sentimientos y pensamientos de poca competencia. 
En términos descriptivos, al evaluar las expectativas de los y las estudiantes, los aspectos donde los y las jóvenes presentan menores expectativas fueron en las dimensiones de relación con compañeros y comparación con la educación presencial. En el primer caso, los y las jóvenes esperan una peor relación en términos personales y comunicación con sus pares en el desarrollo de los cursos en línea durante la pandemia por COVID-19. Este hallazgo es relevante, puesto que, entre las características a considerar en el diseño de los cursos e-learning, está la presencia de interacciones entre estudiantes, que permita el intercambio de información sobre su aprendizaje e información sobre aspectos de tipo socioemocional (Rodrigues, et al., 2019). Así mismo, las expectativas sobre las interacciones entre pares podría ser un obstáculo frente al nivel de motivación de los y las estudiantes durante el desarrollo de estos cursos (Ghavifekr y Mahmood, 2017).

Con respecto a la presencia de menores expectativas en la dimensión de comparación con la educación presencial, es posible identificar que, estas creencias iniciales sobre cómo se desarrollaran la educación virtual durante la pandemia por COVID-19, tiene que ver con la distinción que existen frente a los escenarios de enseñanza remota de emergencia (transición rápida a la educación virtual) y la enseñanza en línea, la diferencia en estos términos surge con la finalidad de establecer un claro contraste con lo que se conoce como educación en línea de calidad, es posible que el aprendizaje en línea producido por la pandemia tenga el estigma de ser de menor calidad que el aprendizaje cara a cara, debido al cambio rápido producido por la pandemia, cuando en verdad, nadie que haga la transición a la enseñanza en línea en estas circunstancias realmente puede diseñar para aprovechar al máximo las ventajas y posibilidades de este formato (Hodges, et al., 2020).

Con respecto a los aspectos con mayor expectativa se encontraron en las dimensiones de autoeficacia para el aprendizaje en línea y la dimensión de docencia en línea. Esto nos indica que los estudiantes en general se sienten preparados para realizar las actividades pedagógicas y administrativas que se presenten en el aula virtual. Al igual que las acciones que pueden realizar sus docentes frente a los procesos de enseñanza y aprendizaje en el entorno virtual durante el tiempo de la pandemia. A nivel general estas creencias se encuentran vinculadas a las características relacionadas con el diseño de los materiales de aprendizaje, el entorno del curso electrónico y la coherencia de los componentes curriculares y finalmente con las competencias por alcanzar al finalizar la formación (Rodrigues, et al., 2019).

Durante el proceso de interacciones para la reorganización factorial de la escala se presentaron las siguientes limitaciones, primero se tuvo como resultado dos ítems que cargaban (aunque en menor medida y por debajo del punto de corte) a un segundo factor y segundo, se generaron dos nuevos factores constituidos por solo dos ítems (evaluación en línea y comparación con la educación presencial), la generación de estos dos factores con pocos ítems originó la presencia de dos dimensiones con menores niveles de fiabilidad. Aunque estos hallazgos son débiles en términos psicométricos, estas dimensiones podrían aportar información importante sobre los elementos comparativos de la modalidad de enseñanza.

La fortaleza de esta investigación reside en la presentación de un cuestionario valido y confiable para la medición de las expectativas de los estudiantes universitarios frente a los escenarios de aprendizaje en línea; no solo durante el tiempo de la pandemia por COVID-19, sino posteriormente a este fenómeno, Poder conocer las expectativas de los universitarios podría contribuir a la detección temprana de estudiantes con riesgo de adaptación y/o menores niveles de compromiso frente a las actividades en líneas ofertadas durante sus cursos, En la medida que los docentes y otros actores educativos puedan conocer sobre las expectativas de los estudiantes, se podrían generar y aplicar medidas preventivas y/o de acompañamiento que beneficien la experiencia académica.

\section{CONCLUSIONES}

A partir de los resultados y discusión se pueden establecer las siguientes conclusiones: 1) El cuestionario CEEVESE, quedo constituido por seis dimensiones y un factor general, en una solución jerárquica; 2) presenta adecuados índices de consistencia interna; reflejando buen nivel de confiabilidad en las respuestas obtenidas por medio de cuestionario; lo que hacen que este cuestionario sea válido y confiable para la medición de este constructo en la Educación Superior chilena; 3) Los estudiantes presentan altas expectativas frente a sus creencias de autoeficacia para el aprendizaje en línea y docencia en línea; 4) se identificaron bajas expectativas frente a la calidad de las relaciones con sus pares.

\section{AGRADECIMIENTOS}

Agradecimientos al Proyecto UCO 1808. Laboratorio de Investigación e Innovación Educativa (IDECLAB) y a la Dirección de Docencia de la Universidad de Concepción para la realización de la presente investigación. 


\section{REFERENCIAS}

Ajzen, I., y Fishbein, M. M., Understanding attitudes and predicting social behavior. En: Englewood cliffs NJ: PrenticeHall, 257-267, New York, USA (1980)

Al-Maroof, R., Al-Qaysi, N., Salloum, S. A., y Al-Emran, M., Blended Learning Acceptance: A Systematic Review of Information Systems Models, https://doi.org/10.1007/s10758-021-09519-0, Technology Knowledge and Learning, 36, 136 (2021)

Al-Rahmi, W., Alias, N. y otros cinco autores, Use of e-learning by university students in Malaysian higher educational institutions: a case in Universiti Teknologi Malaysia, http://doi.org/10.1109/ACCESS.2018.2802325, IEEE Access, 6, 14268-14276 (2018)

Almomani, E. Y., Qablan, A. M., y otros cuatro autores, The Influence of Coronavirus Diseases 2019 (COVID-19) Pandemic and the Quarantine Practices on University Students' Beliefs About the Online Learning Experience in Jordan, https://doi.org/10.3389/fpubh.2020.595874, Frontiers in Public Health, 8, 595874 (2021)

Appiah, M., y Van Tonder, F., E-Assessment in Higher Education: A Review, International Journal of Business Management y Economic Research, ISSN:2229- 6247, 9(6) 1454-1460 (2018)

Aqueveque, A. M., Mondaca, W. A., y otros cuatro autores., Prácticas evaluativas en educación superior, https://doi.org/10.29393/Pa66-15PEAV60015, Paideia (66), 17-47 (2020)

Bandura, A., On the Functional Properties of Perceived Self-Efficacy Revisited, http://doi.org/10.1177/014920631141060638, J. of Manag., (1), 9-44 (2012)

Cai, Z., Fan, X., y Du, J., Gender and attitudes toward technology use: A meta-analysis, https://doi.org/10.1016/j.compedu.2016.11.003, Computers y Education, 105, 1-13 (2017)

Cicha, K., Rizun, M., Rutecka, P., y Strzelecki, A., COVID-19 and Higher Education: First-Year Students' Expectations toward Distance Learning, https://doi.org/10.3390/su13041889, Sustainability, 13(4), 1-19 (2021)

Crisol-Moya, E., Herrera-Nieves, L., y Montes-Soldado, R., Educación virtual para todos: una revisión sistemática, https://doi.org/10.14201/eks.23448, Education in the Knowledge Society, 21, 1-13 (2020)

Davis, F. D., User acceptance of information technology: system characteristics, user perceptions and behavioral impacts, https://doi.org/10.1006/imms.1993.1022, Int. J. Man. Mach. Stud., 38(3), 475-487 (1993)

De los Ríos, L. R., Tovar, J. A., Calero, B. N. P., y Peña, A. Q., Análisis psicométrico preliminar de la escala perfil de impacto emocional Covid-19 en universitarios peruanos, https://doi.org/10.35756/educaumch.v1i15.135, Educa UMCH, 1(15), 71-80 (2020)

Ghavifekr, S., y Mahmood, H., Factors affecting use of e-learning platform (SPeCTRUM) among University students in Malaysia, https://doi.org/10.1007/s10639-015-9435-z, Educ. Inf. Technol., 22(1), 75-100 (2017)

Goksoy, S., Teacher views on the applicability of mastery learning model in teaching learning process, Eurasian Journal of Educational Research, ISSN-1302-597X, 18(78), 203-218 (2018)

Hamodi, C., López Pastor, V. M., y López Pastor, A. T., Medios, técnicas e instrumentos de evaluación formativa y compartida del aprendizaje en educación superior, Perfiles Educativos, ISSN 0185-2698, 37(147), 146-161 (2015)

Hernández, M., Villarroel, V., y Zambrano, J., Dimensiones e indicadores para la metaevaluación de los aprendizajes: reflexión y propuesta del campo teórico de la evaluación en educación superior, Revista Cubana de Educación Superior, ISSN 0257-4314, 39(2) 1-22 (2020)

Hodges, C., Moore, S., y otros tres autores, The difference between emergency remote teaching and online learning, https://er.educause.edu/, Educause Review, (2020)

$\mathrm{Hu}$, L.., y Bentler, P. M., Cutoff criteria for fit indexes in covariance structure analysis: Conventional criteria versus new alternatives, https://doi.org/10.1080/10705519909540118, Struct. Equ. Modeling, 6(1), 1-55 (1999)

Ilgaz, H., y Gülbahar, Y., A snapshot of online learners: e-Readiness, e-Satisfaction and expectations, https://doi.org/10.19173/irrodl.v16i2.2117, The International Review of Research in Open and Distributed Learning, 16(2), 171-187 (2015)

Imsa-ard, P., Thai university students' perceptions towards the abrupt transition to 'forced'online learning in the COVID19 situation, https://doi.org/10.14456/edkkuj.2020.x, Journal of Education Khon Kaen University, 43(3), 30-44 (2020)

Katsikatsou, M., y Moustaki, I., Pairwise Likelihood Ratio Tests and Model Selection Criteria for Structural Equation Models with Ordinal Variables, https://doi.org/10.1007/s11336-016-9523-Z, Psychometrika, 81(4), 1046-1068 (2016)

Krouglov, A., Some Aspects Of Student Engagement In Formative In-Class Peer Review, European Proceedings of Social and Behavioural Sciences EpSBS (2021)

Lorenzo-Lledó, A., Roig-Vila, R., y Lorenzo, G., Evaluación de los MOOC por estudiantes universitarios desde una perspectiva metodológica, https://doi.org/10.30827/publicaciones.v48i2.8367, Publicaciones, 48(2), $401-414$ (2018)

Mamani, P. G. R., Morales-García, W. C., White, M., y Marquez-Ruiz, M. S., Propiedades de una escala de preocupación por la COVID-19: análisis exploratorio en una muestra peruana, Medicina Clínica, 155(12), 535-537 (2020) 
McGaghie, W. C., y Harris, I. B., Learning theory foundations of simulation-based mastery learning, Simulation in Healthcare, 13(3S), S15-S20 (2018)

Montero, I., y León, O.G., A guide for naming research studies in Psychology, Int. J. Clin. Health Psychol., ISSN: 16972600, 7(3), 847-862 (2007)

Moore, M.G., Editorial: Three types of interaction, https://doi.org/10.1080/08923648909526659, Am. J. Distance Educ., 3(2), 1-7 (1989)

Osorio-Gómez, L. A., y Duart, J. M., A hybrid approach to university subject learning activities, https://doi.org/10.1111/j.1467-8535.2011.01175.x, Br. J. Educ. Technol., 43(2), 259-271 (2012)

Paechter, M., Maier, B., y Macher, D., Students' expectations of, and experiences in e-learning: Their relation to learning achievements and course satisfaction, https://doi.org/10.1016/j.compedu.2009.08.005, Computers y Education, 54(1), 222-229 (2010)

Ramírez-Anormaliza, R., Sabaté, F., Llinás-Audet, X., y Lordan, O., Aceptación y uso de los sistemas e-learning por estudiantes de grado de ecuador: El caso de una universidad estatal, Intangible Capital, ISSN: 2014-3214, 13(3), 548581 (2017)

Regmi, K., y Jones, L., A systematic review of the factors-enablers and barriers-affecting e-learning in health sciences education, https://doi.org/10.1186/s12909-020-02007-6, BMC Med. Educ., 20, 1-18 (2020)

Revythi, A., y Tselios, N., Extension of technology acceptance model by using system usability scale to assess behavioral intention to use e-learning, https://doi.org/10.1007/s10639-019-09869-4, Educ. Inf. Technol., 24(4), 23412355 (2019)

Rodrigues, H., Almeida, F., Figueiredo, V., y Lopes, S. L., Tracking e-learning through published papers: A systematic review, https://doi.org/10.1016/j.compedu.2019.03.007, Computers y Education, 136, 87-98 (2019)

Roig-Vila, R., Pais, M. R., y Mellado, M. B., Expectativas de estudiantes universitarios frente a sus capacidades y competencias para participar en cursos abiertos y en línea, Revista de Educación a Distancia (RED), ISSN: 1578-7680, 40, 1-23 (2015)

Salvat, B. G., La evolución del e-learning: del aula virtual a la red, http://dx.doi.org/10.5944/ried.21.2.20577, Revista lberoamericana de Educación a Distancia, 21(2), 69-82 (2018)

Scott, K. C., y Nimon, K., Construct validity of data from a TPACK self-assessment instrument in 2-year public college faculty in the United States, https://doi.org/10.1080/15391523.2020.1790444, Journal of Research on Technology in Education, 53(4), 1-19 (2020)

Suárez Lantarón, B., García-Perales, N., y Elisondo, R. C., La vivencia del alumnado en tiempos COVID-19: estudio comparadoentre las universidades de Extremadura (España) y Nacional de Río Cuarto (Argentina), https://doi.org/10.5944/reec.38.2021.28936, Revista Española de Educación Comparada, 38, 46-48 (2021)

Nieto-Sánchez, Z. C., El e-learning como un recurso de desarrollo educativo, https://doi.org/10.15649/2346030X.485, Aibi Revista de Investigación, Administración e Ingeniería, 6(2), 1-1, (2018)

Santamaría, J. S., y Calvo, S. M., Docencia universitaria con apoyo de entornos virtuales de aprendizaje (EVA), Digital Education Review, ISSN 2013-9144, 21(1), 33-46 (2012)

Velavan, T. P., y Meyer, C. G., The COVID-19 epidemic, https://doi.org/10.1111/tmi.13383, Tropical Medicine and International Health, 25(3), 278-280 (2020)

Wong, S. F., Mahmud, M. M., y Wong, S. S., Effectiveness of Formative E-assessment Procedure: Learning Calculus In Blended Learning Environment, https://doi.org/10.1145/3390525.3390526, Proceedings of the 2020 8th International Conference on Communications and Broadband Networking (2020) 